\title{
Diacronie
}

Studi di Storia Contemporanea

$\mathrm{N}^{\circ} 31,3$ | 2017

"Le armi della politica, la politica delle armi"

\section{Eugenio Di Rienzo, L'Europa e la «Questione napoletana» 1861-1870}

\section{Giuseppe Ferraro}

\section{Q OpenEdition \\ Journals}

\section{Edizione digitale}

URL: http://journals.openedition.org/diacronie/6353

DOI: 10.4000/diacronie.6353

ISSN: 2038-0925

\section{Editore}

Association culturelle Diacronie

\section{Notizia bibliografica digitale}

Giuseppe Ferraro, « Eugenio Di Rienzo, L'Europa e la «Questione napoletana» 1861-1870 », Diacronie [Online], N³1, 3| 2017, documento 9, online dal 29 octobre 2017, consultato il 10 décembre 2020. URL : http://journals.openedition.org/diacronie/6353; DOI : https://doi.org/10.4000/diacronie.6353 


\section{Diacronie}

Studi di Storia Contemporanea

$31,3 / 2017$

"Le armi della politica, la politica delle armi". Ideologie di lotta ed esperienze di guerra

\section{RECENSIONE: Eugenio DI RIENZO, L'Europa e la «Questione napoletana» 1861-1870, Nocera Superiore, D'Amico Editore, 2016, $160 \mathrm{pp}$.}

A cura di Giuseppe FERRARO

Per citare questo articolo:

FERRARO, Giuseppe, «RECENSIONE: Eugenio DI RIENZO, L'Europa e la «Questione napoletana» 1861-1870, Nocera Superiore, D'Amico Editore, 2016, 160 pp.», Diacronie. Studi di Storia Contemporanea : "Le armi della politica, la politica delle armi". Ideologie di lotta ed esperienze di guerra, 31, 3/2017, 29/10/2017,

URL: <http://www.studistorici.com/2017/10/29/ferraro_numero_31/ >

Diacronie Studi di Storia Contemporanea $\rightarrow \underline{\text { http://www.diacronie.it }}$ Rivista storica online. Uscita trimestrale.

redazione.diacronie@hotmail.it

Comitato di direzione: Naor Ben-Yehoyada - João Fábio Bertonha - Christopher Denis-Delacour - Maximiliano Fuentes Codera Anders Granås Kjøstvedt - John Paul Newman - Deborah Paci - Niccolò Pianciola - Spyridon Ploumidis - Wilko Graf Von Hardenberg

Comitato di redazione: Jacopo Bassi - Luca Bufarale - Gianluca Canè - Fausto Pietrancosta - Alessandro Salvador - Matteo Tomasoni Diritti: gli articoli di Diacronie. Studi di Storia Contemporanea sono pubblicati sotto licenza Creative Commons 3.0. Possono essere

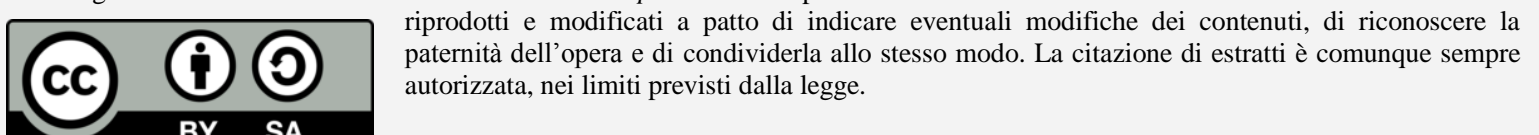




\title{
9/ RECENSIONE: Eugenio DI RIENZO, L'Europa e la «Questione napoletana» 1861-1870, Nocera Superiore, D'Amico Editore, 2016, $160 \mathrm{pp}$.
}

\author{
A cura di Giuseppe FERRARO
}

Eugenio Di Rienzo nel suo ultimo lavoro L'Europa e la «Questione napoletana» tratta alcuni temi di fondamentale importanza per quanto riguarda il processo di unificazione italiana ${ }^{1}$. L'utilizzo di una fonte di prima mano, come il discorso di Lord Lennox alla Camera dei comuni del maggio del 1863, e una puntuale bibliografia, permettono all'autore di studiare l'evoluzione dell'idea di Nazione napoletana prima e dopo il 1860, l'attività del governo napoletano in esilio (formato a Roma da Francesco II), la non trascurabile consistenza di un sentimento nazionale napoletano, le posizioni critiche che assunse la diplomazia internazionale nei confronti delle scelte dei primi governi unitari soprattutto in riferimento ai duri metodi di repressione ${ }^{2}$ messi in atto nelle province meridionali, ma anche di analizzare come molti temi relativi alla questione napoletana sopravvissero negli autori che tennero a «battesimo la nascita della 'questione meridionale'» ${ }^{3}$. È evidente nel libro la capacità dell'autore di intrecciare il punto di vista "interno" con quello "esterno" per conferire alle sue tesi chiarezza e accuratezza. Un libro che, come lo stesso autore sottolinea, è stato scritto in continuità con Il Regno delle Due Sicilie e le Potenze europee 4 .

Le province meridionali al momento dell'unificazione presentavano un maggiore livello di arretratezza dovuto alla mancanza di riforme da parte delle classi dirigenti borboniche ${ }^{5}$, un

\footnotetext{
${ }^{1}$ SCIROCCO, Alfonso, Il Mezzogiorno nella crisi dell'Unificazione (1860-1861), Napoli, S.E.N., 1981; ID., Governo e paese nel Mezzogiorno nella crisi dell'Unificazione (1860-61), Milano, Giuffrè, 1960; ID., Il Mezzogiorno nell'Italia unita (1861-1865), Napoli, Società editrice napoletana, 1979.

${ }^{2}$ Cfr. LATINI, Carlotta, Governare l'emergenza. Delega legislativa e pieni poteri in Italia tra Otto e Novecento, Milano, Giuffrè, 2005, pp. 121-140; SCIROCCO, Alfonso, Amministrazione della giustizia e poteri di polizia prima e dopo l'unità, in Atti del 52.mo Congresso nazionale dell'Istituto per la Storia del Risorgimento italiano, Roma, Istituto per la storia del Risorgimento italiano, 1984; MARTUCCI, Roberto, «La regola è l'eccezione: la legge Pica nel suo contesto", in Nuova Rivista Storica, vol. XCVII, II, 2013, pp. 405-443; ID., Emergenza e tutela dell'ordine pubblico nell'Italia liberale. Regime eccezionale e leggi per la repressione dei reati di brigantaggio (1861-1865), Bologna, Il Mulino, 1980. Si veda anche VIOLANTE, Luciano, «La repressione del dissenso politico nell'Italia liberale: stati d'assedio e giustizia militare», in Rivista di storia contemporanea, V, 4/1976, pp. 481-524.

${ }^{3}$ DI RIENZO, Eugenio, L'Europa e la «Questione napoletana»1861-1870, Nocera Superiore, D’Amico Editore, 2016, p. 77.

${ }^{4}$ ID., Il Regno delle Due Sicilie e le Potenze europee 1830-1861, Soveria Mannelli, Rubbettino, 2012.

${ }^{5}$ Per la situazione sociale, economica e politica prima dell'unità cfr. DE LORENZO, Renata, Borbonia felix. Il
} 
immobilismo che alla fine si era dimostrato letale per la sopravvivenza della stessa monarchia. Questo non significava che non esistessero in limitate porzioni del Regno poli di dinamismo economico, ma rimanevano isolati e dovevano fare i conti con l'assenza di strade e di ferrovie e con bassi livelli di industrializzazione. A questo si aggiungeva una politica borbonica repressiva e poliziesca nei confronti di politici e intellettuali considerati pericolosi per le loro idee riformiste. Tutti elementi che tornarono utili per denigrare ampiamente prima Ferdinando II e poi il figlio Francesco II in seno alle grandi potenze europee. La «leyenda nigra» di Lord Gladstone rappresentava l'esempio certamente più significativo, e finì col dare un'immagine assai negativa e non sempre obiettiva del Regno dei Borbone. Alle critiche, basate su valutazioni spesso indirette di Gladstone, mancava uno sguardo comparativo con le altre realtà politiche italiane preunitarie. Infatti, se la situazione del Regno delle Due Sicilie non era rosea, almeno fino al 1848, quella degli altri Stati italiani non era migliore: «Se, dunque, nel 1861, non c'era una "Borbonia felix", certamente non esisteva nessun Piemonte, nessuna Liguria, nessuna Lombardia, nessuna Toscana "felix" ${ }^{6}$. Ancora nel 1862 un attento osservatore settentrionale, molto vicino al blocco moderato allora al governo (Romualdo Bonfadini), in una lettera, rimarcava come i lamenti che i prefetti facevano in riferimento alle province meridionali erano «troppo consoni con quelli che s'odono in tutte le altre parti d'Italia». E continuava asserendo che, escluso il brigantaggio, geograficamente delimitato alle ex province meridionali, nel resto d'Italia non si stava «né meglio, né peggio delle altre provincie, colla differenza che da noi [al nord] le popolazioni sono più sode e che quanto v'era di già costituito resiste meglio alla dissoluzione che quanto è ancora da costituirsi [...]» ${ }^{7}$.

La situazione nelle province meridionali nei primi anni dello Stato unitario non andò migliorando. Ma certo non si può nemmeno parlare, per il periodo precedente all'unità, di un «primato economico» della Nazione napoletana, e poi, dopo il 1861, di "lager dei Savoia", di genocidio del Sud da parte dei piemontesi, di "scippo" o colonizzazione. Va anche chiarito, come Di Rienzo riesce a fare nel suo volume, che i metodi messi in campo dai governi di Torino e di Firenze per stabilizzare le province meridionali, rafforzare l'unità appena raggiunta, debellare il brigantaggio, non furono certamente meno illiberali di quelli dei Borbone. Di Rienzo infatti sottolinea che se da una parte non è convincente parlare di una realtà meridionale con primati

Regno delle Due Sicilie alla vigilia del crollo, Roma, Salerno editrice, 2013, in particolare pp. 102 et seq. Si veda anche l'indagine sulla scomparsa dei regni preunitari di MACRY, Paolo (a cura di), Quando crolla lo Stato: studi sull'Italia preunitaria, Napoli, Liguori, 2003 (in particolare: Appunti per una fenomenologia del crollo, pp. 3-24); ID., Unità a mezzogiorno. Come l'Italia ha messo assieme i pezzi, Bologna, Il Mulino, 2012; SPAGNOLETTI, Angelantonio, Storia del Regno delle Due Sicilie, Bologna, Il Mulino, 1997, in particolare pp. 271-306; MERIGGI, Marco, Gli stati italiani prima dell'Unità. Una storia istituzionale, Bologna, Il Mulino, 2002; BARONE, Giuseppe, Quando crolla lo stato e non nasce la nazione. Il Mezzogiorno nel Risorgimento italiano, in ROCCUCCI, Adriano (a cura di), La costruzione dello Stato-nazione in Italia, Roma, Viella, 2012.

${ }^{6}$ DI RIENZO, Eugenio, L'Europa e la «Questione napoletana», cit., p. 66.

${ }^{7}$ FERRARO, Giuseppe, Il prefetto e i briganti. La Calabria e l'unificazione italiana, Milano, Mondadori-Le Monnier, 2016, p. 121. 
economici, di «lager dei Savoia», di "genocidio del Sud», non bisogna nemmeno dimenticare «lo stato di arretratezza economica che attanagliava l'intera Penisola e non solo i domini borbonici, la prigionia dura e infamante alla quale furono sottoposti soldati e ufficiali che avevano lealmente seguito Francesco II nell'ultima resistenza e gli spietati metodi di contro-guerriglia, [...]»" D'altronde gli stessi funzionari settentrionali mandati nelle province meridionali mettevano in rilievo da una parte le criticità dei territori annessi, dall'altra l'incapacità del governo centrale di attuare interventi e riforme per migliorare la situazione. Anzi, qualcuno significativamente evidenziava (forse aveva ben in mente gli scritti di Gladstone!) che la politica estera dell'Italia era necessaria farla proponendo un'immagine il più possibile positiva di come si erano risolti i problemi interni nelle province degli ex Stati italiani. Nel 1862 il prefetto della provincia di Cosenza (Calabria Citra), il valtellinese Enrico Guicciardi, sottolineava infatti a Emilio Visconti Venosta che la politica estera si doveva «fare più specialmente coll'amministrazione interna», perché questa poteva dare migliore immagine del nuovo Stato da poco unificato nel contesto internazionale?.

Nelle province napoletane si sviluppò un forte e ampio movimento di resistenza al nuovo ordine statale sotto i Savoia, che al suo interno aveva varie anime, con prospettive e aspirazioni spesso diverse e contrastanti ${ }^{10}$. Era anche sintomatico della presenza, seppur minoritaria, come ricorda Di Rienzo, di un sentimento nazionale napoletano vivo non solo tra le masse contadine e il proletariato, ma anche nel ceto civile, la classe colta, l'esercito e la burocrazia. Il governo napoletano in esilio, presieduto da Pietro Calà Ulloa, sperava però di utilizzare questo sentimento nazionale per ritornare a Napoli, ma «il tentativo degli ultimi sostenitori di Francesco II di avviare un processo di "nazionalizzazione della causa borbonica", che avrebbe dovuto assicurare all'esule di Gaeta la possibilità di riconquistare i suoi domini, arrivò troppo tardi e forse con non sufficiente convinzione fu patrocinato dall'ultimo re di Napoli per conseguire l'esito sperato»" ${ }^{11}$. Anche perché in molti intellettuali e politici in esilio a Gaeta «l'attaccamento alla patria napoletana non era scisso da quello tributato alla più grande patria italiana» ${ }^{12}$.

Dal 1861 il governo borbonico in esilio e i suoi sostenitori cercarono infatti di colpire il nuovo assetto politico creatosi in Italia sul piano diplomatico, ripetendo un meccanismo che aveva fortemente delegittimato nei decenni precedenti sul piano internazionale il Regno delle Due Sicilie. Per sintetizzare, si voleva dimostrare come con i nuovi governi di Torino e di Firenze non

\footnotetext{
${ }^{8}$ DI RIENZO, Eugenio, L'Europa e la «Questione napoletana», cit., p. 7.

${ }^{9}$ Ibidem, p. 124

${ }^{10}$ Cfr. COLAPIETRA, Raffaele, Il brigantaggio postunitario in Abruzzo, Molise e Capitanata; BARRA, Francesco, Il brigantaggio in Campania; PEDIO, Tommaso, Reazioni e brigantaggio in Basilicata (1860-1861) pubblicati tutti in «Archivio storico per le province napoletane», 1983 [ma 1985]. Per la Calabria rimando a GAUDIOSO, Francesco, Calabria ribelle. Brigantaggio e sistemi repressivi nel cosentino (1860-1870), Milano, Franco Angeli, 1987.

${ }^{11}$ DI RIENZO, Eugenio, L'Europa e la «Questione napoletana», cit., pp. 16-17.

${ }^{12}$ Ibidem, p. 17
} 
solo le cose non fossero cambiate ma addirittura peggiorate, come indicavano alcuni dati economici e gli abusi e gli eccessi messi in campo dall'esercito per ripristinare l'ordine pubblico. Una «tattica propagandistica che si mostrerà vincente proprio presso l'opinione politica del Regno Unito», ma anche in Francia e in altre cancellerie europee ${ }^{13}$, anche se non sortirà gli effetti sperati. Sarà proprio la House of Lords ad ospitare numerosi interventi in favore di Francesco II e di altri sovrani spodestati dai piemontesi (nel libro in appendice è stato trascritto il discorso alla Camera dei Comuni di Lord Lennox dell'8 maggio 1863 che richiama bene queste dinamiche). In quelle discussioni emergeva la volontà, da parte di alcuni esponenti politici inglesi, di dimostrare come l'Inghilterra avesse preferito al «despotism of a Bourbon» lo «pseudo-liberalism of a Victor Emmanuel». Alla miopia politico-diplomatica da parte inglese si era aggiunta successivamente anche quella economica, come sottolineava Lord Lennox nel suo discorso. L'unificazione, infatti, secondo i critici del governo italiano, aveva fatto perdere a Londra un sicuro partner commerciale quale era stato prima del 1861 il Regno delle Due Sicilie.

In Francia, come evidenzia Di Rienzo nel volume, la politica propagandistica a favore dei Borbone cercò invece di legare la questione napoletana a quella delle nazioni vittime di oppressione da parte di altri Stati. Il Mémoire di Garnier cercava di assimilare «la rivoluzione dei sudditi di Francesco II contro il governo di Torino all'insurrezione del popolo polacco per emanciparsi dal dominio dell'autocrazia zarista che era scoppiata con grande violenza a Varsavia nella notte del 23 gennaio $1863 »^{14}$. L'avversione verso l'Italia unificata sotto la corona sabauda aveva trovato anche sostegno nel «partito dell'Imperatrice» e nel consiglio privato di Napoleone III. Calà Ulloa presumeva che Napoleone, se nelle province dell'ex regno borbonico non fosse tornata la tranquillità, sarebbe prontamente intervenuto «per modificare la sistemazione politica che si era determinata oltre le Alpi» ${ }^{15}$.

Sebbene la nuova patria italiana fosse sembrata a molti sudditi di Francesco II «matrigna», con l'aumento dell'imposizione fiscale, la leva obbligatoria, la dura repressione, il patriottismo napoletano sbandierato in ritardo dai legittimisti borbonici era destinato a soccombere. Era mosso infatti più da rancore e nazionalismo di rivalsa che da un vero progetto identitario e politico; inoltre i cambiamenti che stavano interessando il quadro politico internazionale in quegli anni (come la vittoria della Prussia contro l'Impero asburgico e la presa di Roma) spinsero lo stesso Francesco II a sciogliere il governo in esilio e poi trasferirsi a Parigi. Il volume evidenzia anche come l'azione diplomatica dei Borbone in esilio di contrastare la nascita del Regno d'Italia e facilitare la rinascita del Regno delle Due Sicilie, anche se destinata a fallire, dimostrasse l'intraprendenza di quei settori costituzionalisti, liberali, federalisti che troppo in ritardo furono

\footnotetext{
${ }^{13}$ Ibidem, p. 25

${ }^{14}$ Ibidem, p. 66.

${ }^{15}$ Ibidem, p. 70.
} 
sostenuti dalla corte borbonica, ma erano capaci di spostare il tema della Nazione napoletana «dallo scenario italiano a quello europeo, per porlo al centro del dibattito internazionale» ${ }^{16}$.

Il libro, in conclusione, permette di riflettere in maniera seria sugli errori commessi da parte delle classi dirigenti liberali nel processo di costruzione dello Stato italiano, senza cercare però per forza un'eccezionalità del caso (e tenendo conto delle differenze), rilevando che, anche in altri processi di costruzione dello Stato nazionale, la violenza del fuoco e dell'acciaio accompagnò e seguì la realizzazione del progetto politico: non dimenticando o tacendo che il 1860 per una parte degli Italiani fu anche l'anno della «sconfitta per mano straniera, della perdita della sovranità economica e politica, del peggioramento delle loro condizioni di vita, dell'inizio del ludibrio cui li espose un sentimento anti-meridionale di chiaro stampo razzista» ${ }^{17}$.

\footnotetext{
${ }^{16}$ Ibidem, p. 24.

${ }^{17}$ Ibidem, pp. 7-8.
} 


\section{L'AUTORE}

Giuseppe FERRARO, dottore di ricerca in Storia contemporanea (Università della Repubblica di San Marino), come cultore della materia svolge la sua attività presso l'Università della Calabria. La sua tesi di dottorato ha vinto due premi nazionali “Pier Paolo D’Attorre" e "Spadolini-Nuova Antologia 2016". È anche membro del comitato scientifico dell'Istituto calabrese per la storia dell'antifascismo e dell'Italia contemporanea e redattore della rivista "Giornale di storia contemporanea».

URL: < http://www.studistorici.com/progett/autori/\#Ferraro > 